\title{
Differentiated Granulocytic Sarcoma
}

National Cancer Institute

\section{Source}

National Cancer Institute. Differentiated Granulocytic Sarcoma. NCI Thesaurus. Code C35819.

A granulocytic sarcoma composed of promyelocytes and more mature neutrophils (WHO 2001). --2003 